\title{
THALASSIOTHRIX SPATHULATA HASLE (THALASSIONEMATACEAE, BACILLARIOPHYCEAE): PRIMER REGISTRO PARA LA FLORA DIATOMOLOGICA MARINA DE CHILE
}

\author{
THALASSIOTHRIX SPATHULATA HASLE (THALASSIONEMATACEAE, \\ BACILLARIOPHYCEAE): FIRST RECORD FOR THE MARINE DIATOM \\ FLORA OF CHILE
}

\author{
Patricio Rivera \& Fabiola Cruces \\ Departamento de Botánica, Facultad de Ciencias Naturales y Oceanográficas, Universidad de Concepción, \\ Casilla 160-C, Concepción, Chile. privera@udec.cl
}

\begin{abstract}
We report the first finding of Thalassiothrix spathulata in the marine phytoplankton of Chile. The species was common in samples collected between $18^{\circ} 16^{\prime} \mathrm{S}$ and $28^{\circ} 06^{\prime}$ 'S. Principal morphological features are described and illustrated.
\end{abstract}

La presencia de espinas en los márgenes valvares, orientadas hacia el extremo apical, es la principal característica que diferencia al géneroThalassiothrix Cleve \& Grunow (1880) de los restantes géneros de la familia Thalassionemataceae: Thalassionema Grunow ex Mereschkowsky emend. Hallegraeff (1986), Trichotoxon Reid \& Round (1988) yLioloma Hasle (Hasle \& Syvertsen 1996).

Thalassiothrix longissima Cleve \& Grunow y Thalassiothrix antarctica Schimper ex Karsten son los únicos taxones de este género señalados hasta la fecha para las aguas marinas chilenas. La primera especie ha sido citada desde los 1940'S hasta los $34^{\circ} 59^{\prime} \mathrm{S}$, en tanto que $T$. antarctica sólo para las aguas de la zona central de Chile (Rivera 1983; Rivera et al. 1990). De acuerdo con la revisión de la familia Thalassionemataceae realizada por Hasle (2001), otras especies de Thalassiothrix señaladas para Chile han sido transferidas a distintos géneros: T. acuta Karsten a Lioloma elongatum (Grunow) Hasle, T. delicatula Cupp a Lioloma delicatulum (Cupp) Hasle y T. frauenfeldii (Grunow) Grunow a Thalassionema frauenfeldii (Grunow) Hallegraeff. Recientemente hemos establecido que este último taxón no está presente en las aguas chilenas, puesto que se le ha confundido conThalassionema bacillare (Heiden) Kolbe y con individuos de gran tamaño de
Thalassionema nitzschioides (Grunow) Mereschkowsky (Rivera et al., datos no publicados).

En esta comunicación informamos sobre el hallazgo de Thalassiothrix spathulata Hasle en aguas chilenas del Pacífico Suroriental. El material estudiado se encuentra depositado en la Colección Diatomológica de la Universidad de Concepción (DIAT-CONC). Numerosos ejemplares de la especie fueron analizados mediante técnicas de microscopía fotónica y electrónica de barrido.

T. spathulata fue común en todas las muestras estudiadas, pero generalmente los frústulos estaban quebrados. En individuos completos el eje apical, levemente curvado, varió entre 800 y $1385 \mu \mathrm{m}$. Esta especie no forma colonias y sus valvas son claramente heteropolares, adelgazándose gradualmente desde el centro hacia los extremos (Figs. 1-2). El extremo apical mide de 3,5 a 3,6 $\mu \mathrm{m}$ de ancho y externamente presenta dos espinas de gran tamaño (Figs. 3, 6-7). El extremo basal es más ancho pudiendo alcanzar hasta $5,5 \mu \mathrm{m}$, es de forma espatulada, más o menos puntiaguda (Figs. 2, 5, 8-9), aunque en algunos casos es obtusamente redondeado (Fig. 10). Las aréolas se disponen en los márgenes de las valvas (11-15 en $10 \mu \mathrm{m}$ ) dejando un sternum ancho (Figs. 3-4, 811), y se distribuyen irregularmente en el extremo basal (Figs. 9-10) y en el extremo apical (Fig. 6). 
Las espinas marginales varían entre 11 y 15 en 100 $\mu \mathrm{m}$ (Figs. 4, 7, 11). Existe un proceso labiado en cada extremo cuya ubicación es visible con el microscopio fotónico. En el extremo apical se sitúa en la parte media de la valva en la base de las espinas (Fig. 3); internamente se orienta en forma oblicua
(Fig. 7) y externamente presenta una abertura circular (Fig. 6). En el extremo opuesto el proceso labiado se ubica cerca del margen valvar, desplazado del extremo mismo a una distancia variable (Figs. 9-10); los labios internos también se orientan de forma oblicua y la abertura externa es circular (Fig. 8).

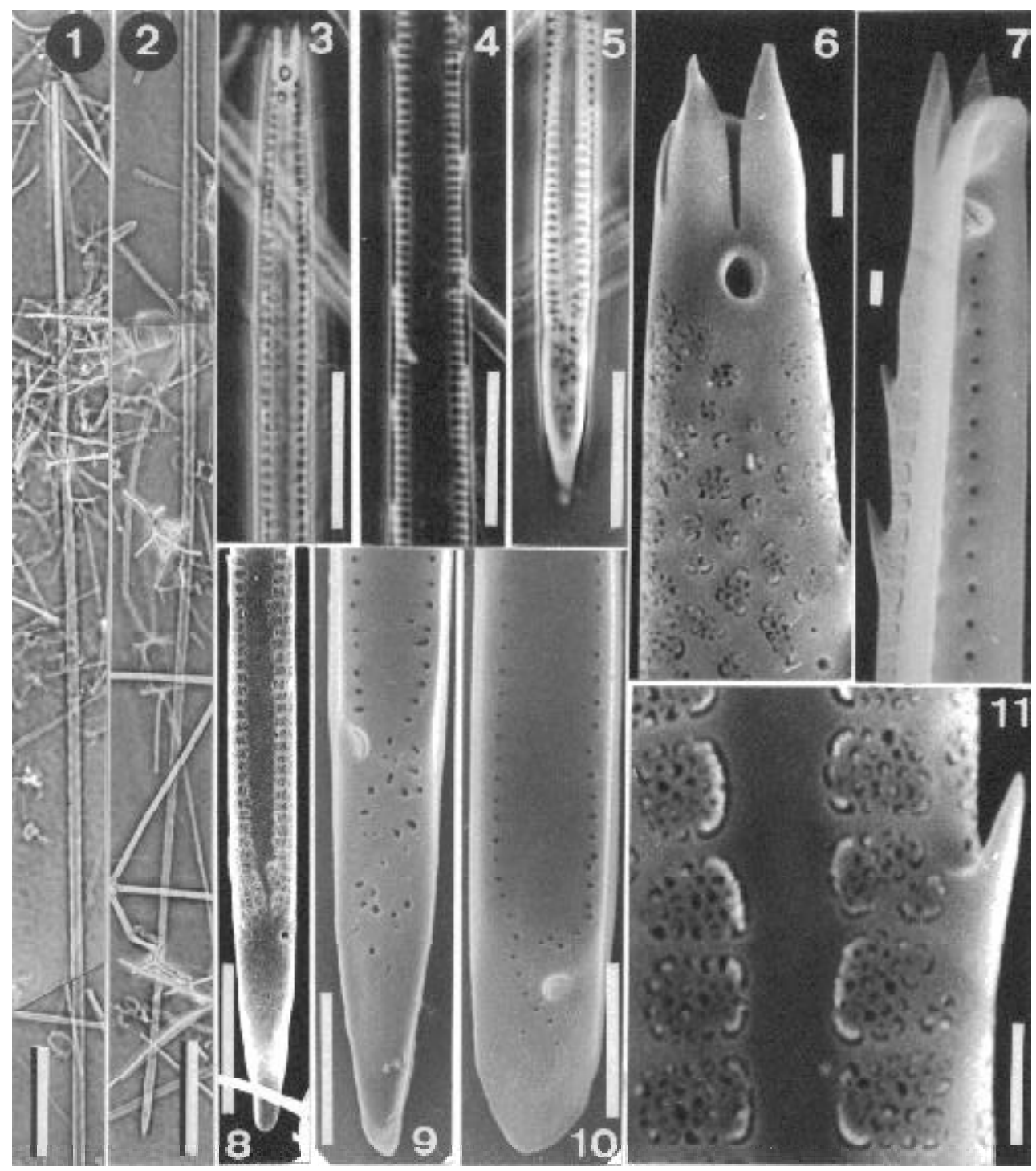

Figuras 1-11. Thalassiothrix spathulata Hasle. Figs. 1-5, ML; Figs. 6-11, MEB. Escala: $50 \mu \mathrm{m}$ (Figs. 1-2), $10 \mu \mathrm{m}$ (Figs. 3-5, 8), $5 \mu \mathrm{m}$ (Figs. 9-10), $1 \mu \mathrm{m}$ (Figs. 6-7, 11). Figs. 1-2. Dos mitades de una misma valva; eje apical algo curvado. Fig. 3. Extremo apical. Fig. 4. Parte media de la valva. Fig. 5. Extremo basal. Fig. 6. Vista externa del extremo apical que lleva dos espinas de gran tamaño y la abertura del proceso labiado; aréolas distribuidas irregularmente. Fig. 7. Vista interna del extremo apical; proceso labiado orientado oblicuamente. Fig. 8. Vista externa del extremo basal con forma puntiaguda, espatulada; la abertura externa del proceso labiado es evidente. Fig. 9. Vista interna del extremo basal; proceso labiado oblicuo, marginal, ubicado lejos del extremo. Fig. 10. Extremo basal obtusamente redondeado. Fig. 11. Vista externa de aréolas y sternum. (ML: microscopía fotónica. MEB: microscopía electrónica de barrido). 
T. gibberula Hasle comparte conT. spathulata algunas características morfológicas y su área de distribución, lo que puede dificultar la correcta identificación de ambos taxones. Sin embargo, $T$. gibberula presenta un mayor número de espinas marginales, el extremo basal no tiene forma espatulada y el proceso labiado no es de posición marginal.

T. spathulata fue descrita originalmente para el Golfo de Tailandia (Hasle 1999) y se le considera una especie típica de aguas tropicales y subtropicales (Hasle 1999, 2001). Ha sido señalada previamente para el Pacífico Suroriental frente a las costas de Ecuador y Perú hasta los $11^{\circ} 19^{\prime}$ 'S (Hasle 2001). En el presente estudio hemos encontrado a $T$. spathulata en diversas muestras de fitoplancton recolectadas

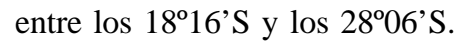

MATERIAL DE ESTUDIO: DIAT-CONC 2322, 18¹6'S, 7447'O, Mar Chile 8, Est. 13, 19.8.1972; DIAT-CONC 3613, $18^{\circ} 20^{\prime} \mathrm{S}, 70^{\circ} 41^{\prime} \mathrm{O}$, Mar Chile 8, Est. $3^{\text {a }}$, 16.8.1972; DIAT-CONC 3619, $18^{\circ} 20^{\prime} \mathrm{S}$, $71^{\circ} 07^{\prime}$ O, Mar Chile 8, Est. 5, 17.8.1972; DIAT-

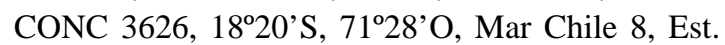
6, 17.8.1972; DIAT-CONC 3715, 18²0'S, 74¹6'O, Mar Chile 8, Est. 12, 18.8.1972; DIAT-CONC 2188, 2349'S, 70³3'O, Mar Chile 8, Est. 34, 25.8.1972; DIAT-CONC 2189, 234' $\mathrm{S}$, 7048'O, Mar Chile 8, Est. 32, 24.8.1972; DIAT-CONC 2190, 2349'S, 7053'O, Mar Chile 8, Est. 31, 24.8.1972; DIAT-

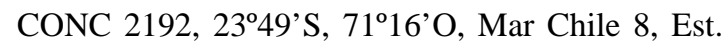
30, 24.8.1972; DIAT-CONC 2191, 235'ㅇ's, 7042'O, Mar Chile 8, Est. 33, 25.8.1972; DIAT-

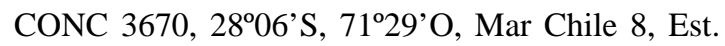
37, 30.8.1972.

\section{AGRADECIMIENTOS}

Agradecemos la colaboración del personal del Laboratorio de Microscopía Electrónica de la Universidad de Concepción, en especial a los señores Raúl Alarcón, Hugo Pacheco y Julio Pugin.

\section{BIBLIOGRAFIA}

Cleve, P.T. \& A. Grunow. 1880. Beiträge zur Kenntniss der arctischen Diatomeen. Kongliga Svenska Vetenskaps-Akademiens Handlingar 17: 1-121.

HALlegraEFF, G.M. 1986. Taxonomy and morphology of the marine plankton diatoms Thalassionema and Thalassiothrix. Diatom Research 1: 57-80.

HasLE, G.R. 1999. Thalassionema synedriforme comb. nov. and Thalassiothrix spathulata sp. nov., two marine, planktonic diatoms from warm waters. Phycologia 38: 54-59.

HASLE, G.R. 2001. The marine, planktonic diatom Family Thalassionemataceae: morphology, taxonomy and distribution. Diatom Research 16: 1-82.

Hasle, G.R. \& E.E. Syvertsen. 1996. Marine Diatoms. In: Identifying Marine Diatoms and Dinoflagellates (ed C.R. Tomas), p. 5-385, Academic Press, San Diego.

ReID, F.M.H. \& F.E. Round. 1988. The antarctic diatom Synedra reinboldii: Taxonomy, ecology and transference to a new genus, Trichotoxon. Diatom Research 2: 219-227.

RIVERA, P. 1983. A Guide for References and Distribution for the Class Bacillariophyceae in Chile between $18^{\circ} 28^{\prime} \mathrm{S}$ and $58^{\circ} \mathrm{S}$. Bibliotheca Diatomologica, J. Cramer, Vaduz, Lichtenstein, Band 3, 386 pp.

Rivera, P., M. Gebauer \& H.L. Barrales. 1990. A Guide for References and Distribution for the Class Bacillariophyceae in Chile between $18^{\circ} 28^{\prime} \mathrm{S}$ and $58^{\circ}$ S. Part II. Data from 1982 to 1988. Gayana Botanica 46: 155-198. 